\title{
The Clinical Findings Useful for Driving Safety Advice for Parkinson's Disease Patients
}

\author{
Rina Ando ${ }^{1}$, Hirotaka Iwaki ${ }^{1,2}$, Tomoaki Tsujii ${ }^{1}$, Masahiro Nagai ${ }^{1}$, Noriko Nishikawa ${ }^{1}$, \\ Hayato Yabe ${ }^{1}$, Ikuko Aiba ${ }^{3}$, Kazuko Hasegawa ${ }^{4}$, Yoshio Tsuboi ${ }^{5}$, Masashi Aoki ${ }^{6}$, \\ Kenji Nakashima ${ }^{7}$ and Masahiro Nomoto ${ }^{1}$; \\ on behalf of the Parkinson's Disease Safe Driving Study Group of Japan
}

\begin{abstract}
:
Objective We conducted a study to obtain information that could be used to provide Parkinson's disease (PD) patients with appropriate advice on safe driving.

Methods Consecutive PD patients who visited our office were studied. Among these patients, those who had experienced driving after being diagnosed with PD were interviewed by neurologists and a trained nurse to investigate their previous car accidents, motor function, cognitive function, sleepiness, levodopa equivalent dose (LED), and emotional dysregulation. The rates of major car accidents before and after the onset of PD were compared.

Results Fifteen patients had experienced a major car accident resulting in human injury or serious property damage since the onset of PD. When the rates of major car accidents before and after the onset of PD were compared, the ratio was 4.3 [95\% confidence interval (CI) 1.9-9.7]. The incidence of accidents after the onset of PD was correlated with age, disease duration, LED, the cognitive function Mini-Mental Scale Examination (MMSE), Japanese translation of the Montreal Cognitive Assessment (MoCA-J), but not the motor symptom score [Unified Pankinson's disease rating scale (UPDRS) part III at the time of the study]. The Questionnaire for Impulsive-Compulsive Disorders in Parkinson's Disease (QUIP) score was also higher in patients with major car accidents.

Conclusion The severity of symptoms (Hoehn-Yahr classification), cognitive function, and disease duration were expected to be risk factors for car accidents. However, the motor symptom score (UPDRS part III) was not associated with the incidence of major car accidents. In addition to a low cognitive function and the severity of symptoms, the QUIP score might be an independent factor that can be referenced when advising PD patients to refrain from driving.
\end{abstract}

Key words: Parkinson's disease, driving, safety, car accident, advice to refrain from driving

(Intern Med 57: 1977-1982, 2018)

(DOI: 10.2169/internalmedicine.9653-17)

\section{Introduction}

Previous studies have shown that driving a car requires cognitive and psychological abilities with motor skills, and that driving capability decreases according to the progression of Parkinson's disease (PD), even in patients with mildto-moderate PD (1-3). Nonetheless, many PD patients still need to drive to get to work or hospital. Previous studies have evaluated the driving skills of PD patients by on-road

\footnotetext{
${ }^{1}$ Department of Neurology and Clinical Pharmacology, Ehime University Graduate School of Medicine, Japan, ${ }^{2}$ Department of Biostatistics, Harvard T.H. Chan School of Public Health, USA, ${ }^{3}$ Department of Neurology, Higashi Nagoya Hospital, Japan, ${ }^{4}$ Department of Neurology, Sagamihara National Hospital, Japan, ${ }^{5}$ Department of Neurology, Fukuoka University, Japan, ${ }^{6}$ Department of Neurology, Tohoku University School of Medicine, Japan and ${ }^{7}$ Department of Neurology, Tottori University Faculty of Medicine, Japan

Received: June 15, 2017; Accepted: October 24, 2017; Advance Publication by J-STAGE: February 28, 2018

Correspondence to Dr. Masahiro Nomoto, nomoto@m.ehime-u.ac.jp
} 
Table 1. Demographic and Clinical Characteristics of the Study Population.

\begin{tabular}{|c|c|c|c|c|c|c|}
\hline \multirow[t]{2}{*}{ Parameter } & \multicolumn{6}{|c|}{ Mean \pm SD (range) or $\mathrm{n}(\%)$} \\
\hline & $\begin{array}{c}\text { Total } \\
(n=140)\end{array}$ & $\begin{array}{c}\text { No car accident } \\
\text { Group A } \\
(n=109)\end{array}$ & $\begin{array}{l}\text { Minor car accident } \\
\text { Group B } \\
(n=16)\end{array}$ & $\begin{array}{c}\text { Major car accident } \\
\text { Group C } \\
(n=15)\end{array}$ & $\begin{array}{c}\text { ANOVA } \\
\text { (All group) }\end{array}$ & $\begin{array}{c}\text { t-test } \\
\text { (Group } \\
\text { A vs. C) }\end{array}$ \\
\hline Number, male & $74(66)$ & $55(54)$ & $8(8)$ & $11(4)$ & & \\
\hline Age, years & $66.8 \pm 8.7(42-87)$ & $67.0 \pm 8.5(42-87)$ & $66.3 \pm 8.3(57-87)$ & $65.9 \pm 10.5(45-83)$ & 0.866 & 0.633 \\
\hline Disease duration, years & $6.8 \pm 4.5(0.7-23.5)$ & $6.3 \pm 4.3(0.7-23.5)$ & $5.9 \pm 2.9(1.4-13.2)$ & $10.2 \pm 5.6(3.1-23.3)$ & $0.006^{*}$ & $0.008 *$ \\
\hline Driving duration, years & $40.8 \pm 10.4(13-67)$ & $41.0 \pm 10.5(18-67)$ & $39.3 \pm 6.3(25-53)$ & $40.6 \pm 12.6(13-62)$ & 0.713 & 0.413 \\
\hline L-dopa, mg/day & $\begin{array}{c}359.8 \pm 155.9 \\
(0-1,050)\end{array}$ & $\begin{array}{c}346.2 \pm 140.9 \\
(0-850)\end{array}$ & $\begin{array}{c}351.7 \pm 109.0 \\
(200-650)\end{array}$ & $\begin{array}{c}463.3 \pm 236.3 \\
(250-1,050)\end{array}$ & $0.023 *$ & $0.006^{*}$ \\
\hline Total LED, mg/day & $\begin{array}{c}551.0 \pm 308.6 \\
(0-1,860)\end{array}$ & $\begin{array}{c}511.0 \pm 255.9 \\
(0-1,410)\end{array}$ & $\begin{array}{l}520.7 \pm 247.1 \\
(300-1,115)\end{array}$ & $\begin{array}{c}871.5 \pm 479.7 \\
(250-1,860)\end{array}$ & $<0.001 *$ & $<0.001 *$ \\
\hline H\&Y classification & $2.6 \pm 0.77(1-5)$ & $2.5 \pm 0.8(1-5)$ & $2.6 \pm 6.7(1-3.5)$ & $3.2 \pm 0.7(2.5-4)$ & $0.011 *$ & $0.003 *$ \\
\hline \multicolumn{7}{|l|}{ UPDRS } \\
\hline Part 1 & $1.5 \pm 1.9(0-8)$ & $1.4 \pm 1.8(0-8)$ & $1.5 \pm 1.4(0-4)$ & $2.3 \pm 2.4(1-8)$ & 0.178 & 0.063 \\
\hline Part 2 & $8.0 \pm 6.4(0-34)$ & $7.5 \pm 6.3(0-34)$ & $6.6 \pm 3.7(3-14)$ & $13 \pm 7.2(3-28)$ & $0.005^{*}$ & $0.005^{*}$ \\
\hline Part 3 & $26.4 \pm 11.1(1-64)$ & $25.9 \pm 11.1(1-64)$ & $25.8 \pm 10.2(10-45)$ & $30.5 \pm 10.9(8-51)$ & 0.322 & 0.245 \\
\hline Part 4 & $1.5 \pm 2.2(0-12)$ & $1.3 \pm 2.0(0-12)$ & $1.0 \pm 1.5(0-5)$ & $3.3 \pm 2.9(0-9)$ & $0.002 *$ & $0.002 *$ \\
\hline MoCA-J & $22.9 \pm 4.0(10-30)$ & $23.0 \pm 3.9(13-30)$ & $23.1 \pm 3.5(14-27)$ & $21.9 \pm 4.8(10-28)$ & 0.649 & 0.447 \\
\hline MMSE & $27.7 \pm 2.9(11-30)$ & $27.9 \pm 2.3(21-30)$ & $27.8 \pm 1.8(24-30)$ & $25.8 \pm 5.9(11-30)$ & $0.039 *$ & $0.011 *$ \\
\hline QUIP & $0.37 \pm 0.9(0-4)$ & $0.27 \pm 0.7(0-4)$ & $0.5 \pm 1.2(0-4)$ & $1.0 \pm 1.2(0-3)$ & $0.017 *$ & $0.005^{*}$ \\
\hline ESS & $5.0 \pm 3.7(0-19)$ & $4.7 \pm 3.6(0-19)$ & $5.9 \pm 4.8(0-19)$ & $6.1 \pm 2.9(3-10)$ & 0.207 & 0.150 \\
\hline
\end{tabular}

$* \mathrm{p}<0.05$ for ANOVA and $<0.025$ for t-test

$†$ Patients who never had car accidents after PD onset. Of the 82 patients still driving, 28 (34.1\%) drove $\geq 5$ days/week, 26 (32.7\%) >1-4 days/week, and 28 $(34.1 \%) 1$ day/week.

$\$$ Patients who had car accidents resulting in minor property damage after PD onset. Two patients had 2 accidents, 1 had 4 accidents, and 13 had 1 accident. Of the 12 patients still driving drive, 9 (75\%) drove $\geq 5$ days/week, $1(8 \%)>1-4$ days/week, and $2(17 \%) 1$ day/week.

$\S$ Patients who had car accidents resulting in injury to the patient, passengers or others involved in the accident, or resulting in serious property damage. Type of accident: rear end accident, run against the wall or guardrail, or error in judgment at an intersection. There are no one patient who repeated major car accidents. Of the 11 patients still driving, 4 (36.3\%) drove $\geq 5$ days/week, 2 (18.1\%)>1-4 days/week, and 5 (45.5\%) 1 day/week.

ESS: Epworth sleep scale, H\&Y: Hoehn-Yahr, LED: L-dopa equivalent dose, MMSE: Mini-Mental Scale Examination, MoCA-J: Japanese translation of the Montreal Cognitive Assessment, QUIP: Questionnaire for Impulsive-Compulsive Disorders in Parkinson's Disease, UPDRS: Unified Parkinson's disease rating scal

testing or with the use of a driving simulator (4-7). However, there is little information on the data that can be obtained in a regular office visit that can be used when giving driving advice to PD patients. In a previous study using a driving simulator, we found that handling errors increased with postural instability (1). In the current study, we interviewed PD patients about their past accidents and examined their motor and cognitive ability at regular outpatient visits to obtain information that could be useful for advising patients on safe driving.

\section{Materials and Methods}

We screened consecutive PD patients who were diagnosed according to the UK Brain Bank criteria and who visited Ehime University Hospital from August 1, 2014 to October 31 , 2015. Those who were currently driving or who had stopped driving after the onset of PD were enrolled as the study population. Interviews were conducted by neurologists and a specially appointed nurse. The following items were evaluated: age, gender, Hoehn-Yahr (H\&Y) severity classification, Unified Parkinson's Disease Rating Scale (UPDRS) score, Japanese translation of the Montreal Cognitive As- sessment (MoCA-J) score, Mini-Mental Scale Examination (MMSE) score, Questionnaire for Impulsive-Compulsive Disorders in Parkinson's Disease (QUIP) score (8), Epworth sleep scale (ESS) score, and drug history at the time of the study. The MoCA-J and MMSE scores both evaluate the cognitive function on a 30-point scale; the MoCA-J score better reflects the frontal lobe function than the MMSE score. The following items concerning the driving status were also evaluated: driving history (period of time in years between the onset of PD and the interview date or the onset of PD and the date on which the patient stopped driving), history of driving accidents, injuries caused by accidents (to self or others), and sleepiness or sudden loss of consciousness at the time of an accident. We analyzed those data related to car accidents. Furthermore the rate of major car accidents before and after the onset of PD was studied. The SAS JMP software program (v10) was used to perform the statistical analyses. Two-tailed $\mathrm{p}$ values of $<0.05$ were considered to indicate statistical significance.

\section{Results}

Two hundred forty-one of the 325 PD patients had a driv- 

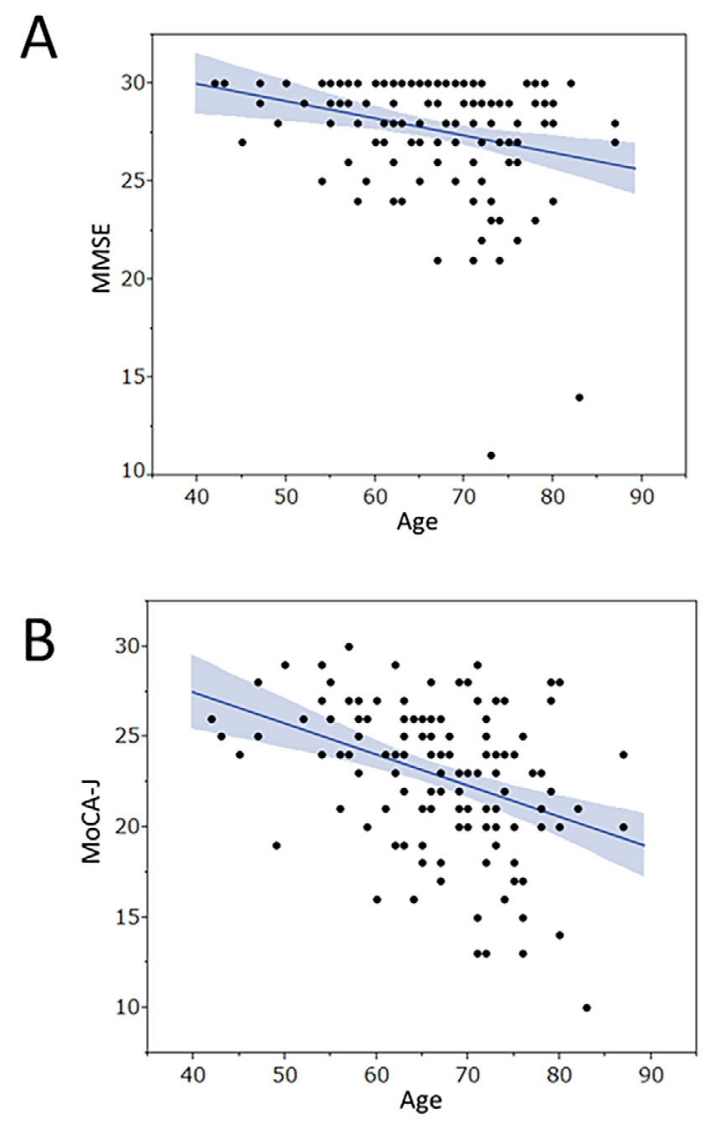

Figure 1. The MMSE (A) and MoCA-J (B) scores decreased according to age (ANOVA $\mathrm{p}=\mathbf{0 . 0 0 1 9}$ and $\mathrm{p}<0.001$, respectively). The line denotes the estimated regression and the shadow shows the $95 \%$ CI of its slope.

ing license. Among these, 140 PD patients (male, $n=74$; female, $\mathrm{n}=66$ ) who were currently driving or who had stopped driving after the onset of PD were enrolled in the study (Table 1). Thirty-four patients were no longer driving at the time of the study. The mean time from the onset of PD until the patient stopped driving was 5.7 years (range 0-17.8 years). Seventeen patients had an MMSE score of $\leq 24$ points, and 33 had a MoCA-J score of $\leq 20$ points. Both the MMSE and MoCA-J scores decreased significantly with age (Fig. 1A and B, respectively). When applied a univariate linear regression model was applied, both MMSE and MoCA-J scores were negatively correlated with age $(\mathrm{p}=0.0019$ and $\mathrm{p}$ $<0.001$, respectively). The ESS scores increased significantly with the disease duration (Fig. 2). The mean MoCA-J score of 22.9 points was lower than the mean MMSE score of 27.7 points $(\mathrm{p}<0.0001)$. The results show that MoCA-J score is more sensitive than the MMSE score for measuring the decline of the cognitive function in PD.

Past car accidents were classified according to timing (before or after the onset of PD) and severity (minor or major). Major car accidents were defined as accidents resulting in injury to the patient, passengers, others involved in the accident, or serious property damage. Minor car accidents were defined as accidents resulting in minor property damage.

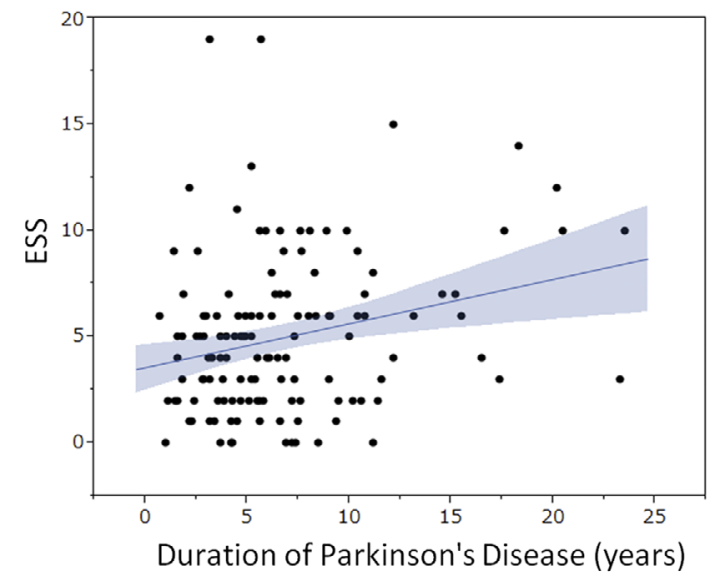

Figure 2. The ESS scores increased according to the duration of PD $(\mathbf{p}<0.0001)$. The line denotes the estimated regression and the shadow shows the $95 \%$ CI of its slope.

Sixteen patients (11\%) had experienced a major accident before the onset of PD and 15 patients (11\%) had experienced a major accident after the onset of PD. Twenty-six patients (19\%) had experienced a minor accident before the onset of PD and 16 patients (11\%) had experienced a minor accident after the onset of PD. The mean duration of driving history was 34.9 years before the onset of PD and 5.8 years after the onset of PD. The rate of major accidents after the onset of PD was higher than that before the onset of PD $(0.0177$ versus 0.0031 accidents/year/person, respectively). When comparing the rate of major car accidents before and after the onset of PD, the ratio was 4.3 [95\% confidence interval (CI) 1.9-9.7]. Four of the 15 patients who had experienced a major car accident after the onset of PD stated, "I didn't realize what was happening until I had already crashed", indicating that the accident was possibly caused by suddenonset sleep.

Accidents that had occurred after the onset of PD were categorized into three groups: no accident (group A, $\mathrm{n}=109$ ), minor accident (group $\mathrm{B}, \mathrm{n}=16$ ), and major accident (group $\mathrm{C}, \mathrm{n}=15)$ groups. The differences between groups were assessed using the analysis of variance (ANOVA) (Table 1). When the three groups were compared, there was no difference in age or driving history. Additionally, there were no differences in any of the items between groups A and B. In comparison to Group A, Group C had a higher levodopa dose, total levodopa equivalent dose (LED) (9), H\&Y classification, UPDRS part II score, UPDRS part IV score, QUIP score, and MMSE score. (Table 1). Furthermore, among the UPDRS part III scores, there were significant differences in the bradykinesia and postural instability values of the groups, while the overall UPDRS did not differ to a statistically significant extent. When we analyzed the differences among the three groups using the Kruskal-Wallis test, the bradykinesia and postural instability values were found to be significantly different $(\mathrm{p}=0.0119$ and $\mathrm{p}=0.0204$, respectively); the Wilcoxon test also revealed significant differences between Groups A and C ( $\mathrm{p}=0.0034$ and $\mathrm{p}=0.0068$, 
Table 2. Estimated Coefficients of a Logistic Multivariate Model of Clinical Characteristics for Having a Major Car Accident.

\begin{tabular}{lcccl}
\hline & Estimate & Standard error & $\chi^{2}$ & $\mathrm{p}$ value \\
\hline Intercept & 1.53377701 & 4.2292999 & 0.13 & 0.7169 \\
Age & 0.01231868 & 0.0389516 & 0.10 & 0.7518 \\
Disease duration & 0.00241433 & 0.0168305 & 0.02 & 0.8859 \\
Total LED & -0.0025063 & 0.0019178 & 1.71 & 0.1913 \\
H\&Y classification & -0.7092462 & 0.5559468 & 1.63 & 0.2020 \\
MMSE & 0.11863273 & 0.0890112 & 1.78 & 0.1826 \\
QUIP & -0.5778229 & 0.2628645 & 4.83 & $0.0279^{*}$ \\
\hline
\end{tabular}

*Statistically significant $(\mathrm{p}<0.05)$.

H\&Y: Hoehn-Yahr, LED: L-dopa equivalent dose, MMSE: Mini-Mental Scale Examination, QUIP: Questionnaire for Impulsive-Compulsive Disorders in Parkinson's Disease, UPDRS: Unified Parkinson's disease rating scale

respectively).

All of the three patients who received a total LED of $\geq$ $1,500 \mathrm{mg} /$ day at the time of the study and $46 \%$ of the patients with a total LED of $\geq 900 \mathrm{mg} /$ day had experienced a major car accident. Thirty-eight percent of PD patients with grade $\geq 4$ on the H\&Y scale and $17 \%$ of the patients with grade $\geq 3$ had experienced a major car accident. With regard to the QUIP score, $29 \%$ of patients with a score of $\geq 2$ points and $25 \%$ patients with a score of $\geq 1$ had experienced a major car accident. Furthermore, with regard to the MMSE score, $100 \%$ patients with a score of $\leq 20$ and $60 \%$ of patients with a score of $\leq 21$ had experienced a major car accident. These findings indicate that patients with a total LED of $\geq 900 \mathrm{mg} /$ day, an H\&Y classification of $\geq 4$, a QUIP score of $\geq 1$, or an MMSE score of $\leq 20$ were highly likely to have caused a major accident or to do so in the future. We conducted a multivariate analysis using a logistic regression model to predict the probability of a person having a major car accident. The analysis revealed that the QUIP score was not correlated with the cognitive function or the severity of PD symptoms but that it was independently correlated with having had a major car accident (Table 2).

\section{Discussion}

The risk of accidents when driving has been reported to increase when cognitive function, as measured by the MMSE, declines to $\leq 24$ points (10). In PD patients, the deteriorating cognitive function is also associated with a higher rate of driving accidents (11). In addition, PD patients are slower to make decisions and have reduced spatial cognition, which should further reduce their driving skills in novel situations and on busy roads (12). Studies on driving ability and treatment have also been reported (13). In 2014, $\mathrm{Hu}$ et al. (14) evaluated the cognitive function of 486 PD patients and 141 controls using the MMSE and MoCA. They found that although the MMSE scores of PD patients did not change over the long-term course, there was a clear decrease in their MoCA scores. The PD patients in our study had very similar MMSE and MoCA-J scores to the patients in that previous study. The association between a declining cognitive function and driving performance has also been studied. Esser et al. (15) assessed the actual onroad driving performance of patients with neurological disorders and found that the total MoCA score differed significantly between patients who passed and failed a driving test, and that the factor was independent of their neurological disorders. An assessment of the sensitivity and specificity yielded two cutoff MoCA scores: a cutoff score of $<2$ captured $100 \%$ of the patients who failed but only $16.7 \%$ of the patients who passed, while a cutoff score of $>27$ captured $100 \%$ of the patients who passed but only $4.9 \%$ of the patients who failed. In our study, $100 \%$ of the patients with a MoCA-J score of $>28$ had never had an accident and $100 \%$ of the patients with a MoCA-J score $\leq 10$ had experienced a major accident. Even though our investigation was a retrospective interview study, the results were close to those obtained from on-road testing.

The ESS scores of the no accident and major accident groups did not differ to a statistically significant extent. Previous research has also shown that ESS scores and daytime sleepiness were not correlated with driving skill $(4,12,16)$. The reasons for this may be that the driving tests were conducted in the morning when patients were in their best "on" state (12). In our study, 4 of the 15 patients in the major accident group had experienced sudden-onset sleep, which led to an accident in three patients. This indicates that the ESS score does not always predict the incidence of PD-related driving accidents.

Dopamine agonists are associated with a risk of suddenonset sleep; thus, PD patients taking non-ergot alkaloids are warned not to drive. In this cohort, $70 \%$ of the PD patients receiving non-ergot alkaloids continued driving against drug label warnings. However, there were no differences between the patients taking non-ergot alkaloids and those taking ergot alkaloids (17). These findings suggest that if medication counseling is provided, then the incidence of car accidents may not increase.

The driving skill of PD patients has been evaluated in various studies $(2-7,11,12,16,18-21)$. In a study of 104 
PD patients who had a current driver's license and were driving, researchers conducted on-road testing using a checklist and evaluated disease characteristics, motor symptoms, cognitive function, and epidemiological aspects offroad (20). In that study, $65 \%$ of the PD patients passed the on-road test, while $35 \%$ failed. In comparison to the patients who passed, the patients who failed were older, had a longer driving history, and walked less over a 1-year period. The drugs used and ESS score were not associated with accidents within a 5-year period (20). Only one crash was reported in that study. In our study, 15 of 140 patients (11\%) had experienced a major car accident after the onset of PD. The reason for this difference is not clear, but it may be related to the traffic conditions or the methods used to obtain information from patients. Furthermore, in 2015, the National Police Agency of Japan reported that the rates of driving accidents among normal drivers aged in their 60s and 70 s were 0.0052 and 0.0063 accidents/year/person, respectively (22). The rates among PD patients before and after the onset of PD were 0.0031 and 0.0177 , respectively. The rate among $\mathrm{PD}$ patients before the onset of $\mathrm{PD}$ was not higher than that for normal drivers. However, the rate after the onset of PD was higher than that of the normal drivers.

The results of our study are compatible with previous studies on driving among PD patients. However, the present study was associated with some limitations. It should be noted that our study was retrospective in nature. The patients experienced car accidents prior to their clinical examination. Furthermore, although a trained nurse attempted to obtain precise information on the car accidents from the patients and their families, we did not obtain information from the police.

The incidence of car accidents among PD patients was correlated with age, disease duration, cognitive function, and various symptom scores, including the H\&Y classification and UPDRS scores part II, part IV. The UPDRS part III score was not associated with the incidence of major car accidents. It has been reported that neurologists overestimate the ability of PD patients to drive (3). The MMSE was associated with the incidence of major car accidents in this study. Patients with dementia, now undergo memory tests at the renewal of their licenses in order to prevent car accidents. A memory test might be also useful for promoting safe driving among PD patients. The accident rate was particularly high among patients with an H\&Y classification of $\geq 4$ and an MMSE score of $\leq 20$. The QUIP score was correlated with major car accidents, and the correlation was independent of PD symptoms. It is presumed that patients with high QUIP scores are unable to control their mind sufficiently to consider that they should not drive or to drive more carefully despite advice from their family or medical staff. A QUIP score of $\geq 1$ was associated with major car accidents and this information should be taken into consideration when advising patients on driving. Other factors that should be considered include low MMSE scores, and higher levodopa doses, total levodopa equivalent doses, H\&Y clas- sifications, UPDRS part II scores and UPDRS part IV scores.

This was a retrospective study on car accidents and the clinical findings were obtained at the time of this study. There was time difference between these events. The clinical findings, including the cognitive function at the time of study might be useful for advising PD patients on driving safety. However, the results should be confirmed in a largersize study on the driving of PD patients.

The risk of automobile collisions increased after the onset of PD and PD patients should be advised to use alternative transportation. In some areas, however, a patient may need to drive in order to maintain their life, especially in rural areas. Automobiles with collision prevention devices have been developed and PD patients who need to continue driving should be advised to use such cars.

The protocol was approved by the Ehime University Hospital Research Ethics Committee.

Written informed consent was obtained from the patients prior to participation in this study.

\section{Author's disclosure of potential Conflicts of Interest (COI).}

Masahiro Nagai: Honoraria, Novartis. Masashi Aoki: Honoraria, Mitsubishi Tanabe Pharma; Research funding, Pfizer. Masahiro Nomoto: Honoraria, Dainippon-Sumitomo, Hisamitsu and Kyowa Hakko Kirin; Research funding, Shionogi.

\section{Financial Support}

This study was supported by Grants-in-Aid from the Ministry of Health, Labour and Welfare of Japan and the Japan Research Foundation for Clinical Pharmacology.

\section{Acknowledgement}

We appreciated the contribution of Mrs Chieko Yamazaki for her endeavor during interviews with patients and their caregivers for this study.

\section{References}

1. Thiri Kyaw W, Nishikawa N, Moritoyo T, et al. Evaluating the driving ability in patients with Parkinson's disease using a driving simulator. Intern Med 52: 871-876, 2013.

2. Uc EY, Rizzo M, Anderson SW, Sparks J, Rodnitzky RL, Dawson JD. Impaired visual search in drivers with Parkinson's disease. Ann Neurol 60: 407-413, 2006.

3. Heikkilä VM, Turkka J, Korpelainen J, Kallanranta T; Summala. Decreased driving ability in people with Parkinson's disease. J Neurol Neurosurg Psychiatry 64: 325-330, 1998.

4. Crizzle AM, Classen S, Uc EY. Parkinson disease and driving: an evidence-based review. Neurology 79: 2067-2074, 2012.

5. Devos H, Ranchet M, Emmanuel Akinwuntan A, Uc EY. Establishing an evidence-base framework for driving rehabilitation in Parkinson's disease: a systematic review of on-road driving studies. NeuroRehabilitation 37: 35-52, 2015.

6. Vardaki S, Devos H, Beratis I, Yannis G, Papageorgiou SG. Exploring the association between working memory and driving performance in Parkinson's disease. Traffic Inj Prev 17: 359-366, 2016.

7. Madeley P, Hulley JL, Wildgust H, Mindham RH. Parkinson's dis- 
ease and driving ability. J Neurol Neurosurg Psychiatry 53: 580$582,1990$.

8. Tanaka K, Wada-Isoe K, Nakashita S, Yamamoto M. Impulsive compulsive behaviors in Japanese Parkinson's disease patients and utility of the Japanese version of the Questionnaire for ImpulsiveCompulsive Disorders in Parkinson's disease. J Neurol Sci 331: 76-80, 2013.

9. Tomlinson CL, Stowe R, Patel S, Rick C, Gray R, Clarke CE. Systematic review of levodopa dose equivalency reporting in Parkinson's disease. Mov Disord 25: 2649-2653, 2010.

10. Iverson DJ, Gronseth GS, Reger MA, Classen S, Dubinsky RM, Rizzo M. Quality Standards Subcommittee of the American Academy of Neurology. Practice parameter update: evaluation and management of driving risk in dementia: report of the Quality Standards Subcommittee of the American Academy of Neurology. Neurology 74: 1316-1324, 2010.

11. Dubinsky RM, Gray C, Husted D, et al. Driving in Parkinson's disease. Neurology 41: 517-520, 1991.

12. Ranchet M, Paire-Ficout L, Uc EY, Bonnard A, Sornette D, Broussolle E. Impact of specific executive functions on driving performance in people with Parkinson's disease. Mov Disord 28: 1941-1948, 2013

13. Tujii T, Kyaw WT, Iwaki H, et al. Evaluation of the effect of pregabalin on simulated driving ability using a driving simulator in healthy male volunteers. Int J Gen Med 7: 103-108, 2014.

14. Hu MT, Szewczyk-Królikowski K, Tomlinson P, et al. Predictors of cognitive impairment in an early stage Parkinson's disease cohort. Mov Disord 29: 351-359, 2014.

15. Esser P, Dent S, Jones C, et al. Utility of the MOCA as a cognitive predictor for fitness to drive. J Neurol Neurosurg Psychiatry
87: 567-568, 2016.

16. Amick MM, D'Abreu A, Moro-de-Casillas ML, Chou KL, Ott BR. Excessive daytime sleepiness and on-road driving performance in patients with Parkinson's disease. J Neurol Sci 252: 13-15, 2007.

17. Ando R, Yamasaki $\mathrm{C}$, Iwaki $\mathrm{H}$, et al. Incidence of severe traffic accidents and medicines in patients with Parkinson's disease. Rinsho Yakuri (Jpn J Clinical Pharmacology and Therapeutics) 48: 167-171, 2017.

18. Classen S, Holmes J. Executive functions and driving in people with Parkinson's disease. Mov Disord 28: 1909-1911, 2013.

19. Stolwyk RJ, Scally KA, Charlton JL, Bradshaw JL, Iansek R, Georgiou-Karistianis N. Self-regulation of driving behavior in people with Parkinson disease. Cogn Behav Neurol 28: 80-91, 2015.

20. Devos H, Vandenberghe W, Tant M, et al. Driving and off-road impairments underlying failure on road testing in Parkinson's disease. Mov Disord 28: 1949-1956, 2013.

21. Classen S, Brumback B, Monahan M, et al. Driving errors in Parkinson's disease: moving closer to predicting on-road outcomes. Am J Occup Ther 68: 77-85, 2014.

22. Annual report of the National Police Agency of Japan on the occurrence of traffic accidents in 2015 (in Japanese). 2016 May 30 [cited 2016 Nov. 3]. Available from: http://www.e-stat.go.jp/SG1/e stat/List.do?lid=000001150496

The Internal Medicine is an Open Access article distributed under the Creative Commons Attribution-NonCommercial-NoDerivatives 4.0 International License. To view the details of this license, please visit (https://creativecommons.org/licenses/ by-nc-nd/4.0/).

(C) 2018 The Japanese Society of Internal Medicine Intern Med 57: 1977-1982, 2018 